\title{
A case report of electrical storm during aortic valve replacement and cardiac artery bypass requiring 16 attempts at electrical cardioversion
}

\author{
Mori T., Kodaka M., Nihei W., Okamura K., Nishiyama K., Komori M. \\ Tokyo Women's Medical University Medical Center East, Dept of Anaesthesiology, Tokyo, Japan
}

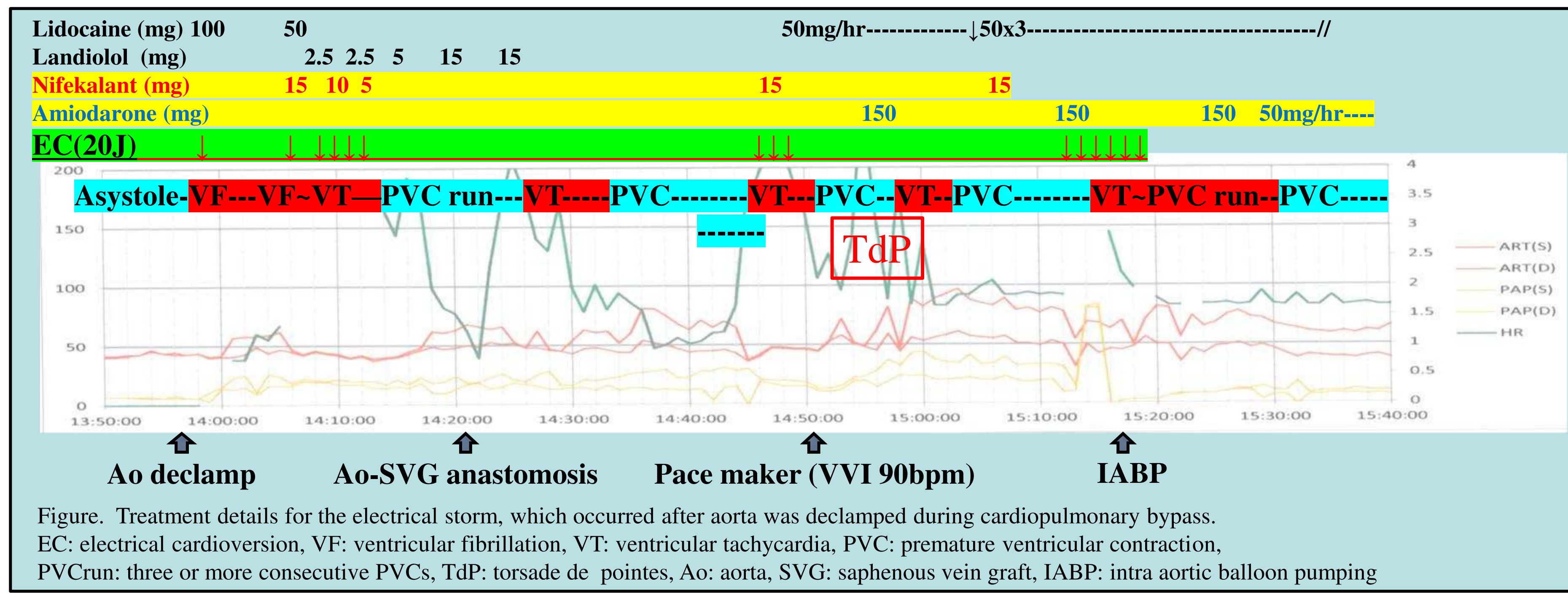

\section{Background}

Nifekalant, a K+ channel blocker (class III agent by Vaughan Williams classification) available only in Japan, is used as often as amiodarone for suppressing electrical storm (ES) signs such as ventricular fibrillation and tachycardia (VF and VT) ${ }^{1)}$. In our department, nifekalant is a first-choice drug in such situations. It has no inotropic effect and a short half-life. Here, we report a case in which we failed to suppress ES with nifekalant and 16 attempts at electrical cardioversion (EC), but subsequently succeeded with amiodarone after cardiopulmonary bypass (CPB) in cardiac surgery.

\section{Case report}

A 65-year-old male, diagnosed with aortic valve stenosis (AS) and angina pectoris, underwent an elective aortic valve replacement (AVR) and coronary artery bypass. His past history included hypertension and 8 years of dialysis treatment.

After starting $\mathrm{CPB}$, cardiac arrest was induced immediately after cardioplegia infusion and maintained with $500-700 \mathrm{ml}$ infusion every 30 minutes. Left internal thoracic artery to left anterior descending artery, saphenous vein graft to right coronary artery anastomosis, and AVR were performed. When the aorta was declamped, VF occurred (Figure). Several attempts at EC and twice administrations of nifekalant $(15 \mathrm{mg})$ with landiolol seemed to be effective, but did not last long. After third administration of nifekalant, the electrocardiogram showed torsade de pointes. Subsequently, we administered magnesium sulfate and amiodarone $(150 \mathrm{mg}) \times 3$ times. This seemed to be effective to stop the ES, which sustained for 80 minutes.

During this period, normothermia was maintained with no electrolyte imbalance, the bypass graft flow was satisfactory, and echocardiography showed only diffuse hypokynesis, which gradually improved toward the weaning of CPB. After ICU admission, no VF or VT occurred with continuous infusion of amiodarone, and the patient was discharged from ICU on the third postoperative day without complication.
Table. Characteristics of nifekalant and amiodarone

\begin{tabular}{|l|cc|}
\hline & Nifekalant & Amiodarone \\
\hline Site of action & Delayed rectifier K channel & Multiple-channel \\
QT prolongation & ++ & + \\
Inotropic effect & None & Negative \\
Half-life & $1.5 \mathrm{~h}-2 \mathrm{~h}$ & 14 days \\
Metabolism and excretion & $30 \%$ renal & $100 \%$ hepatic \\
Approval in Japan & 1999 & 2007 \\
Remarks & Available only in Japan & $\begin{array}{c}\text { First-choice drug } \\
\text { for ES in most } \\
\text { countries }\end{array}$ \\
\hline
\end{tabular}

\section{Discussions}

Nifekalant is an antiarrhythmic agent approved in Japan (table). Though some studies show that nifekalant is not at all inferior to amiodarone in managing patients with fatal ventricular arrhythmias 2,3), there is no large-scale study comparing the efficacy of the two drugs. We experienced a case in which nifekalant was not effective but amiodarone was. The reason is unknown, but an underlying character of nifekalant, a pure $\mathrm{K}+$ channel blocker, could have been less effective than amiodarone, multi-channel blocker in this case.

\section{Learning points}

1. In Japan, nifekalant is used as often as amiodarone in managing patients with fatal ventricular arrhythmias, but there is no large comparative study for effectiveness.

2. We experienced a case in which nifekalant was less effective than amiodarone for ES.

\section{References}

1. Guidelines for Drug Treatment of Arrhythmias (JCS 2009)

2. J Anesth 28: 587-592

3. J Cardiovasc Pharmacol 66: 600-609 